\title{
A Quick Method for Estimating the Propagation Direction of Coronal Mass Ejections Using STEREO-COR1 Images
}

\author{
M. Mierla · J. Davila $\cdot$ W. Thompson - B. Inhester • N. Srivastava · M. Kramar • \\ O.C. St. Cyr • G. Stenborg • R.A. Howard
}

Received: 21 May 2008 / Accepted: 10 September 2008 / Published online: 10 October 2008

(C) Springer Science+Business Media B.V. 2008

\begin{abstract}
We describe here a method to obtain the position of a coronal moving feature in a three-dimensional coordinate system based on height-time measurements applied to
\end{abstract}

M. Mierla (凶)

Royal Observatory of Belgium, Ringlaan 3, 1180 Brussels, Belgium

e-mail: marilena@oma.be

M. Mierla

Astronomical Institute of the Romanian Academy, Str. Cutitul de Argint 5, 040557 Bucharest, Romania

J. Davila · O.C. St. Cyr

NASA Goddard Space Flight Center, Greenbelt, MD 20771, USA

J. Davila

e-mail: josephmdavila@gmail.com

O.C. St. Cyr

e-mail: orville.c.stcyr@nasa.gov

W. Thompson

Adnet Systems, Inc., 164 Rollins Avenue, Suite 303, Rockville, MD 20852, USA

e-mail: william.t.thompson@nasa.gov

B. Inhester · N. Srivastava

Max-Planck-Institut für Sonnensystemforschung, Max-Planck Str. 2, 37191 Katlenburg-Lindau,

Germany

B. Inhester

e-mail: binhest@mps.mpg.de

N. Srivastava

e-mail: nandita@prl.res.in

N. Srivastava

Udaipur Solar Observatory, P.O. Box 198, Badi Road, Udaipur 313001, India

M. Kramar

Catholic University of America, 620 Michigan Avenue, Washington, DC 20064, USA

e-mail: kramar@helio.gsfc.nasa.gov 
STEREO data. By using the height - time diagrams from the two SECCHI-COR1 coronagraphs onboard STEREO, one can easily determine the direction of propagation of a coronal mass ejection (i.e., if the moving plasma is oriented toward or away from the Earth). This method may prove to be a useful tool for space weather forecasting by easily identifying the direction of propagation as well as the real speed of the coronal mass ejections.

Keywords Corona: structures · Coronal mass ejections: initiation and propagation

\section{Introduction}

Coronal mass ejections (CMEs) are a direct consequence of the dynamic nature of the solar atmosphere. They are enormous eruptions of plasma ejected from the Sun into interplanetary space, over the course of minutes to hours. They can create major disturbances in the interplanetary medium and trigger severe magnetic storms when they collide with the Earth's magnetosphere. The LASCO coronagraphs (Brueckner et al., 1995) onboard the SOHO spacecraft have provided unprecedented observations of CMEs. Since coronagraphs measure mainly the photospheric light scattered by free electrons in the corona, giving the integrated density along the line of sight, they only provide a particular view of a CME projected on the plane of the sky. From the LASCO images, it is not possible to infer the true direction of propagation of the CMEs. New data from the Solar Terrestrial Relations $O b$ servatory (STEREO), which was launched in October 2006, provided us with the first-ever stereoscopic images of the Sun's atmosphere. The two STEREO spacecraft A and B orbit the Sun at approximately 1 AU near the ecliptic plane with a slowly increasing angle of about $45^{\circ}$ year between them. The stereoscopic images obtained from the Sun Earth Connection Coronal and Heliospheric Investigation (SECCHI) (Howard et al., 2008) onboard STEREO will help us to determine the location of CMEs in three-dimensional (3D) space and to derive their true direction of propagation.

In the past, coronagraph data have been analyzed by means of height - time (HT) diagrams. HT measurements have been widely used to track moving features in the solar corona. Most of these measurements are obtained by choosing a specific feature in a timelapse movie and tracking its position with time. More sophisticated HT techniques have been developed to automatically detect the faintest moving features in an image (see, e.g., Sheeley et al., 1999). The advantages of the HT technique are its immediate display of motion and speeds and its sensitivity, with which it can pick up the faintest features and detect their motions. The success of this method suggests its potential applicability to STEREO data analysis.

Several techniques have been developed to infer the location of coronal structures in 3D space (Pizzo and Biesecker, 2004; Inhester, 2006; Feng et al., 2007; Aschwanden et al., 2008). Geometric parameters of halo CMEs have been previously measured (Zhao, Plunkett, and Liu, 2002; Michalek, Gopalswamy, and Yashiro, 2003; Xie, Ofman, and Lawrence, 2004; Michalek, 2006) by using a cone model technique applied to LASCO data. In this

\footnotetext{
G. Stenborg

Interferometrics, Inc., 13454 Sunrise Valley Drive, 240, Herndon, VA 20171, USA

e-mail: stenborg@kreutz.nascom.nasa.gov
}

\section{R.A. Howard}

Naval Research Laboratory, Code 7660, 4555 Overlook Ave. SW, Washington, DC 20375, USA

e-mail: russ.howard@nrl.navy.mil 
technique it is assumed that the CMEs propagate almost radially above $2 R_{\odot}$ and that their angular widths remain nearly constant while propagating through corona. In another study, Cremades and Bothmer (2004) derived the geometrical properties of structured CMEs from a set of 124 flux-rope CMEs observed in LASCO-C2 data. Based on this study, Thernisien, Howard, and Vourlidas (2006) developed a forward-modeling technique for flux-rope-like CMEs to reproduce the CMEs morphology. Another technique that has been used to determine the 3D structure of a CME makes use of polarization measurements of the white-light corona (Moran and Davila, 2004; Dere, Wang, and Howard, 2005). In what follows, we present a technique based on HT analysis, which allows us to quickly identify the direction of propagation of the CMEs and to determine their real propagation speed. We apply this technique to the data acquired with SECCHI-COR1 coronagraphs (Thompson et al., 2003) onboard the STEREO A and B spacecraft. A precise analysis of the COR1 data requires that the complex geometric settings be taken into account. These methods are used for loops and small-scale structures on the Sun's surface. CMEs are large-scale and sometimes diffuse structures. The fronts and cores of CMEs can often be localized in the images only with reduced precision. As a consequence, we can lift some of the geometrical constraints and simplify others, hence making the analysis more transparent and faster. In brief, we will work under the simplified assumptions that $i$ ) the distance of the spacecraft off the ecliptic can be neglected and ii) the geometry is affine.

\section{The Instrument and the Observations}

The SECCHI (Howard et al., 2008) is a suite of remote-sensing instruments consisting of an extreme ultraviolet imager, two white-light coronagraphs, and a heliospheric imager. The SECCHI-COR1 coronagraph is a classical Lyot-type internally-occulted coronagraph that observes the white-light corona from $1.4 R_{\odot}$ to $4 R_{\odot}$ (Thompson et al., 2003). The coronagraph includes a linear polarizer, which is used to suppress scattered light and to extract the polarized brightness signal from the solar corona. The detector is an EEV model 42-40 CCD, with $2048 \times 2048$ pixels, $13.5 \mu \mathrm{m}$ on a side. The nominal spatial resolution is 7.5 arcsec (pixel size of 3.75 arcsec). Typically, the COR1 images are $2 \times 2$ binned onboard before telemetering to the ground. The polarized brightness is extracted from three sequential images taken with polarizations of $0^{\circ}, 120^{\circ}$, and $240^{\circ}$. The cadence of a sequence is every 5 or 10 minutes. A monthly background is subtracted from each polarization component to remove the scattered light and the F-corona. These background images are available at the STEREO COR1 Web site. ${ }^{1}$ They are derived from a daily background that is built based on a combination of pixel-by-pixel median and minimum filters. These daily images are then combined over four-week periods to form minimum background images. For this study, we used total brightness images, taken on 15 May, 20 May, and 5 June 2007. Coronal mass ejections were observed on these days at position angles (PA) of around $90^{\circ}, 180^{\circ}$, and $240^{\circ}$, respectively (see Figure 1). To remove the contribution of the streamers and visualize the contribution of the $\mathrm{CME}$ alone, we subtract a minimum model image. The image is created by taking the minimum value in each pixel over the images of the day when the CME was observed. The final images were rotated to align them in the STEREO mission plane (the plane that contains the two spacecraft A and B and the Sun). EUVI disk images have been inserted after coaligning them with the COR1 images. These images taken by

\footnotetext{
${ }^{1}$ http://cor1.gsfc.nasa.gov/data/background/.
} 


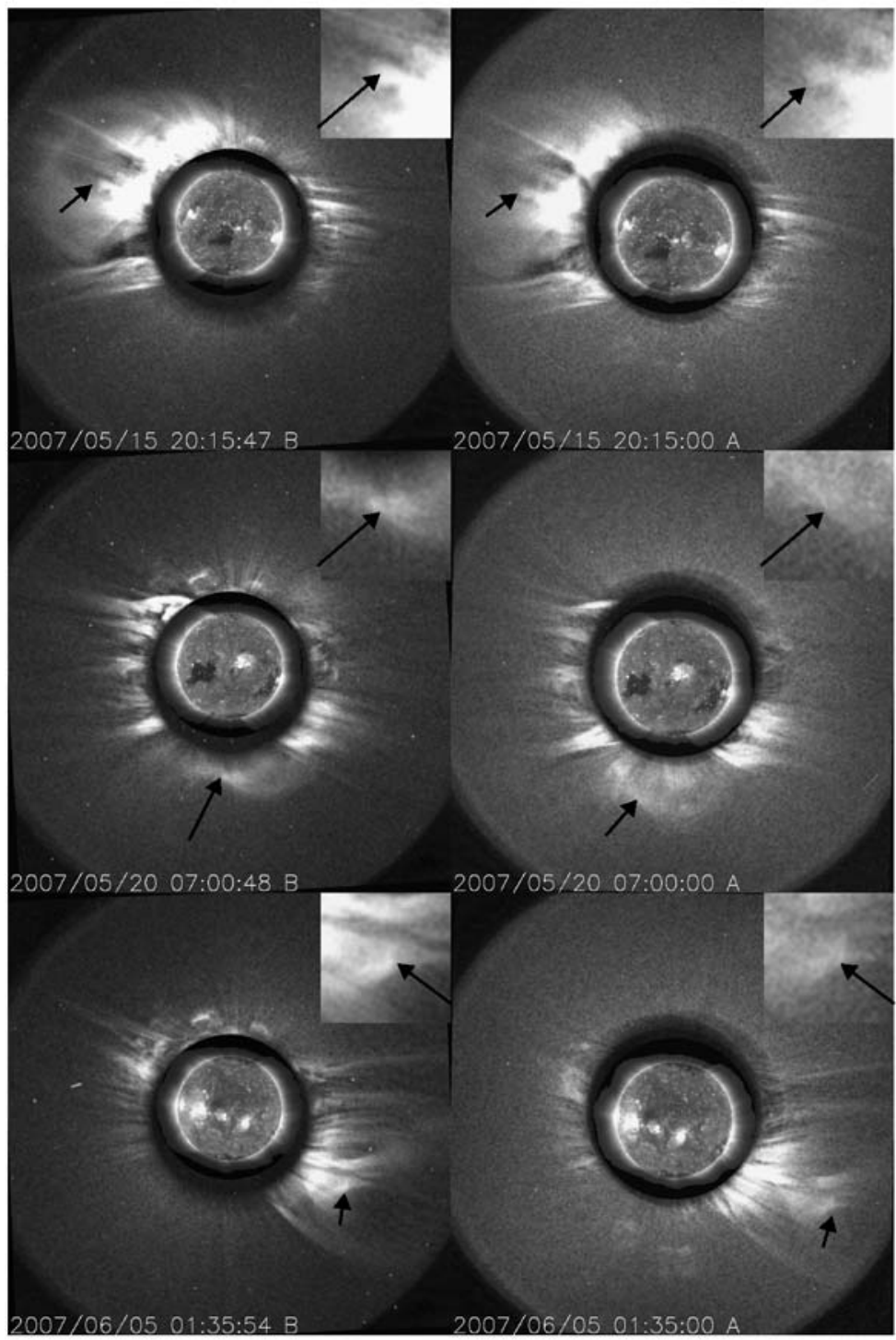

Figure 1 SECCHI-EUVI and SECCHI-COR1 combined images taken on 15 May (upper panels), 20 May (middle panels), and 5 June (lower panels) from both A (right) and B (left) spacecraft. The COR1 images show the white-light total brightness corona from $1.4 R_{\odot}$ to $4 R_{\odot}$ and EUVI images show the UV corona at $19.5 \mathrm{~nm}$. Arrows indicate the traced features. A zoomed region containing the traced feature is shown in the upper right corner of each image.

SECCHI-EUVI (Extreme Ultraviolet Imager) show the UV solar corona in the Fe XII line at $19.5 \mathrm{~nm}$ (corona temperature of around 1.5 MK). The source region of the CME on 20 May was identified as the bright active region at the center of the solar disk (see Figure 1, middle panels). For the other two CMEs, there were no visible changes in EUVI data to infer whether the CMEs were front-sided. 


\section{Method Description}

The two observer positions and any object point to be reconstructed exactly define a plane. By varying the object point, all such planes have in common that they contain the two observer positions. The planes from this set are called epipolar planes (Inhester, 2006). They project on both observer's images as lines, called, accordingly, epipolar lines. The epipolar planes supply a natural coordinate system for stereoscopic reconstruction.

The objects we deal with here are localized blobs of plasma that we can discriminate in a CME cloud. The selection of the features to be tracked was based simply on the fact that they were easily and unambiguously identifiable by naked eye in both A and B images. Because these features are part of the global CMEs their selection will not affect our results regarding the determination of the direction of propagation of the CME. As we have seen in the previous section, the blobs are often still diffuse and cannot be localized to high precision compared to, for example, loops in EUV images. For this reason, we argue that it is justified to relax the reconstruction constraints slightly.

For this study we have assumed that $i$ ) the two spacecraft are in the ecliptic plane (i.e., the STEREO mission plane and the ecliptic are approximately the same) and ii) we can use affine geometry for the reconstruction instead of projective geometry. As a consequence, we can treat epipolar planes in each image as being parallel to the ecliptic.

The first assumption is justified since the orbits of the two spacecraft are very close to the ecliptic plane. Let $z_{\mathrm{scA}}$ and $z_{\mathrm{scB}}$ be their distance off the ecliptic, $d_{\mathrm{AB}}$ be their mutual distance, and $d_{\mathrm{sc}}$ be their distance from the Sun center. From our simplified calculations we will obtain the 3D position of an object in spherical coordinates $\left(R_{3 \mathrm{D}}, \theta, \lambda\right)$ relative to the epipolar plane through the Sun center. We therefore essentially ignore the final rotation of these coordinates into the ecliptic system. The error affects mainly the latitude and longitude angles $\theta$ and $\lambda$ and its magnitude is of the order of the rotation angles between the epipolar and the ecliptic system: $\operatorname{atan} 2\left(z_{\mathrm{scA}}-z_{\mathrm{scB}}\right) / d_{\mathrm{AB}}$ and $\operatorname{atan}\left(z_{\mathrm{scA}}+z_{\mathrm{scB}}\right) / 2 d_{\mathrm{sc}}$. For the cases treated here these angles never exceed $3^{\circ}$. The second assumption affects the reconstruction more fundamentally. In an image, a distance $R$ of an object from the projected center of the Sun measures the angular difference between two view directions. We assume that all objects we see in the coronagraph images are close to the Sun and therefore interpret $R$ as a distance $d_{\mathrm{sc}} \tan R$ from the line of sight to the Sun center. Its real magnitude, however, is $d_{\mathrm{obj}} \tan R$, where $d_{\text {obj }}$ is the object's true distance from the spacecraft. The relative error we commit is therefore $\left(d_{\mathrm{obj}}-d_{\mathrm{sc}}\right) / d_{\mathrm{sc}}$. For an object at $4 R_{\odot}$ in front of the Sun the error amounts to $2 \%$. This error seems tolerable compared to the precision with which we can determine $R$ for plasma blobs in CMEs. This error may, however, increase for the final 3D position since the depth of the object is calculated from the difference of two such distances $R$. For a small base angle $\gamma$ this difference is small and the relative error accordingly enhanced.

From these assumptions and using a simple geometry (Figure 2) we can get the position of the object in spherical coordinates:

$$
\begin{aligned}
R_{3 \mathrm{D}} & =\sqrt{\left(R_{2 \mathrm{D}}\right)^{2}+z^{2}}, \\
\lambda & =\arctan \left[\tan \left(\frac{\gamma}{2}\right) \frac{a-b}{a+b}\right], \\
\theta & =\arctan \frac{R_{2 \mathrm{D}}}{z},
\end{aligned}
$$

where

$$
R_{2 \mathrm{D}}=\sqrt{\alpha^{2}+\beta^{2}+2 \alpha \beta \cos \gamma},
$$



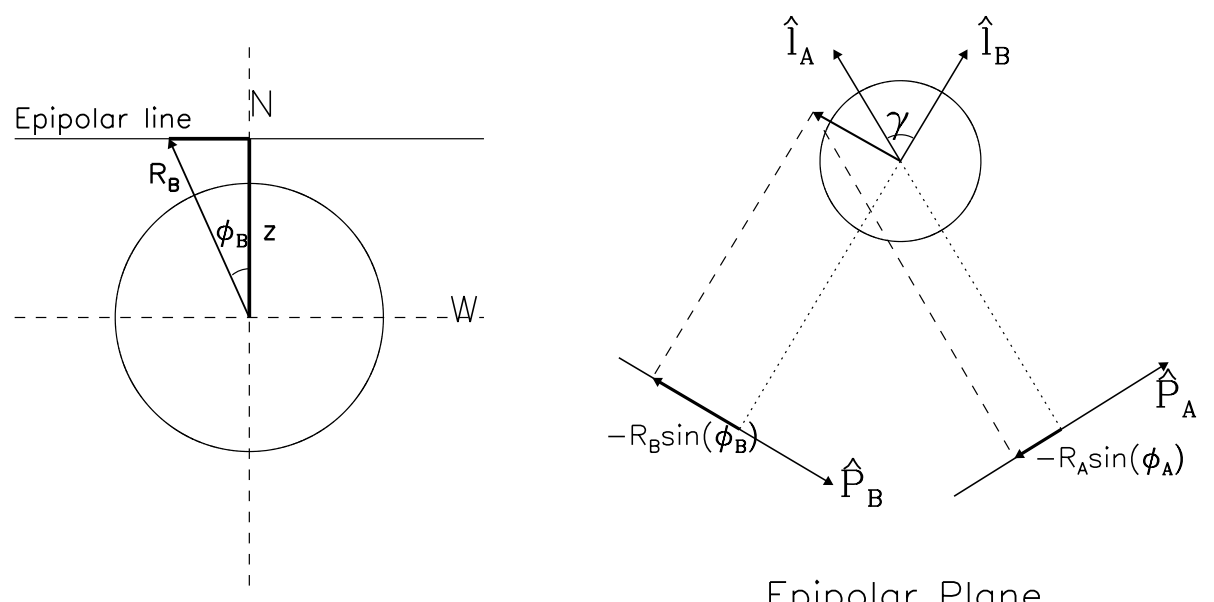

Epipolar Plane

Figure 2 Left: Sketch of the projected position of the moving feature on COR1 B image. The feature is at a distance $R_{\mathrm{B}}$ from the Sun center and its ecliptic position angle is $\phi_{\mathrm{B}}$. The epipolar line is represented by the solid horizontal line. Right: Sketch of the epipolar plane containing the moving feature. The moving feature is represented by the vector ending at the intersection of the two dashed lines. The two COR1 A and $\mathrm{B}$ images are represented by $\hat{\mathbf{P}}_{\mathrm{A}}$ and $\hat{\mathbf{P}}_{\mathrm{B}}$ vectors. $\hat{\mathbf{l}}_{\mathrm{A}}$ and $\hat{\mathbf{l}}_{\mathrm{B}}$ are perpendicular to $\hat{\mathbf{P}}_{\mathrm{A}}$ and $\hat{\mathbf{P}}_{\mathrm{B}}$, respectively. $\gamma$ is the separation angle between the two spacecraft. The circles in the two panels represent the solar disk.

$$
\begin{aligned}
\alpha & =R_{\mathrm{B}} \sin \phi_{\mathrm{B}} / \sin \gamma, \\
\beta & =-R_{\mathrm{A}} \sin \phi_{\mathrm{A}} / \sin \gamma, \\
z & =R_{\mathrm{B}} \cos \phi_{\mathrm{B}}=R_{\mathrm{A}} \cos \phi_{\mathrm{A}}, \\
a & =-R_{\mathrm{A}} \sin \phi_{\mathrm{A}}, \\
b & =R_{\mathrm{B}} \sin \phi_{\mathrm{B}} .
\end{aligned}
$$

$R_{\mathrm{A}}$ is the distance from the projected feature in image A to the Sun center and $\phi_{\mathrm{A}}$ is the ecliptic position angle (EPA) (measured counterclockwise from the north ecliptic pole of each image). The equations are derived in the Appendix.

The geometry on which the equations are based is described in Figure 2. The left panel of Figure 2 represents the view from image plane B. At the top of the image is the North Pole $(\mathrm{N})$ of the ecliptic. A similar geometry is valid for the feature in image A. The right panel of Figure 2 shows the view from the North Pole of the ecliptic. $\hat{\mathbf{l}}_{\mathrm{A}}$ and $\hat{\mathbf{l}}_{\mathrm{B}}$ are unit vectors in the view direction of spacecraft $A$ and $B$. The angle $\gamma$ between $\hat{\mathbf{l}}_{\mathrm{A}}$ and $\hat{\mathbf{l}}_{\mathrm{A}}$ is the separation angle of the two spacecraft.

\section{Application of the Method to the COR1 Data}

Using the equations derived in the Appendix, one can get the position in a $3 \mathrm{D}$ coordinate system of a moving feature. In this way the position of the CME at each instant can be inferred and the direction of propagation can be derived. The real speed can then be calculated by estimating the time derivative of $R_{3 \mathrm{D}}$ (see the Appendix). The HT diagrams here are produced by tracking a particular feature in a sequence of images covering the CME. 
The specific features of these three CMEs are marked in Figure 1 by the arrows. These are plane-of-sky measurements similar to those produced in the past by using coronagraph data (Srivastava et al. (2000), Yashiro et al. (2004), and references therein). The EPA of these features is also measured for each point, at a given time. The HT diagrams are shown in Figure 3. The left panel of Figure 3 shows the height (upper plot) and the ecliptic position angle (lower plot) versus time for the CME on 15 May. The moving feature is observed at a EPA of around $80^{\circ}$ on both $\mathrm{A}$ and $\mathrm{B}$ images. Note that the distance of the identified feature from the Sun center (measured in solar radii) is slightly larger in A as compared with B. The middle panel of Figure 3 shows the HT plot for a point on the leading edge of the CME observed on 20 May. The point could be easily identified in both A and B images because of its particular shape (see Figure 1, middle panels). The CME is observed below the south pole of the Sun (EPA around $180^{\circ}$ ). Its distance from the Sun center is the same on A and B images, unlike the first event. However, A and B differ in the EPA. The right panel in Figure 3 shows the HT for the CME on 5 June, at the southwest limb of the Sun (EPA around $240^{\circ}$ ). In this case the distance measured in the STEREO A image is larger than for STEREO B. Using these HT plots and the knowledge of the ecliptic position angle of the location of the CME, we can infer whether the tracked moving feature is part of a front-side or a back-side CME. The features tracked in our work to make the corresponding HT diagrams are different from those selected by Yashiro et al. (2004). They tracked the fastest structures in CMEs whereas we concentrate in those features that could be identified in both images. Nevertheless, their analyses were performed on LASCO data, so only the projected parameters could be determined. Note that this analysis (of a specific structure of the CME) will not give any information about the entire CME (since particular parts of the CME could have different speeds; see, e.g., Low and Hundhausen, 1987), and we may not be able to derive general properties from these events.

\section{Discussion and Conclusion}

Figure 4 shows plots of the distance from the Sun center $\left(R_{3 \mathrm{D}}\right)$ (upper panels), the ecliptic longitude (middle panels), and the ecliptic latitude (lower panels) versus time for the three tracked features. The ecliptic longitude is measured with respect to the median plane of the two spacecraft (i.e., the plane that contains the midpoint between the two spacecraft; see the Appendix). The value of the longitude ranges between $-180^{\circ}$ and $180^{\circ}$. This also means front-side disk longitude ranges between $-90^{\circ}$ and $90^{\circ}$. We see that for the $\mathrm{CME}$ on 15 May, the longitude is around $-70^{\circ}$, meaning that the event is observed on the visible part of the solar disk, close to the east limb. The distance from the Sun center of the CME core is observed in the range between $2.4 R_{\odot}$ and $3.2 R_{\odot}$. The latitude was estimated to be around $14^{\circ}$. The slight temporal changes in longitude and latitude (i.e., the feature's motion toward the central meridian and away from the ecliptic) suggest that the magnetic field of the surrounding regions is strong enough with a configuration such that can deviate the moving plasma (at least at the heights under consideration, i.e., up to $3 R_{\odot}$ ). The deflection of the CMEs from the radial direction has been also observed in the past (see, e.g., St. Cyr et al. (1999) for MLSO and SMM data and Cremades and Bothmer (2004) for LASCO data). The CME on 20 May is observed at a ecliptic longitude of around $-2^{\circ}$, meaning that it is a front-side CME. The latitude is around $-30^{\circ}$ (south of the ecliptic). The leading edge of this CME could be tracked up to a distance of $6 R_{\odot}$. In this distance range the ecliptic longitude is constant. The CME on 5 June is a back-side CME (with a longitude of around $132^{\circ}$ ), observed on the west side of the Sun. The constant values of the ecliptic longitude 


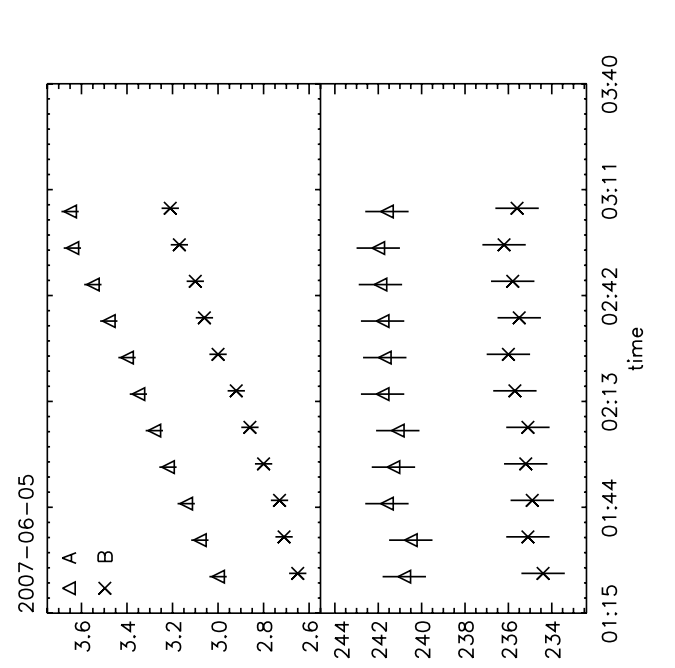

뫃

둥

of

크

홍

क :

를

¿

乎

$\therefore \ddot{n}$

气

江

$\Xi$ 일

点总

플

흐 망

롱

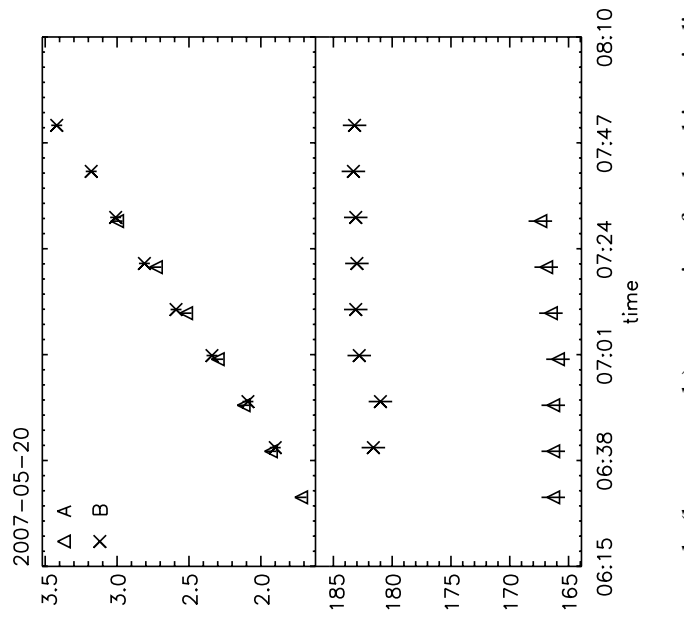

$\Xi . \Xi$

过.

○

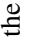

흔

¿

总。

号总

$>$.

क

ฝี ญ

¿

3ㅇํㅇ

0.

恶总

응

कू

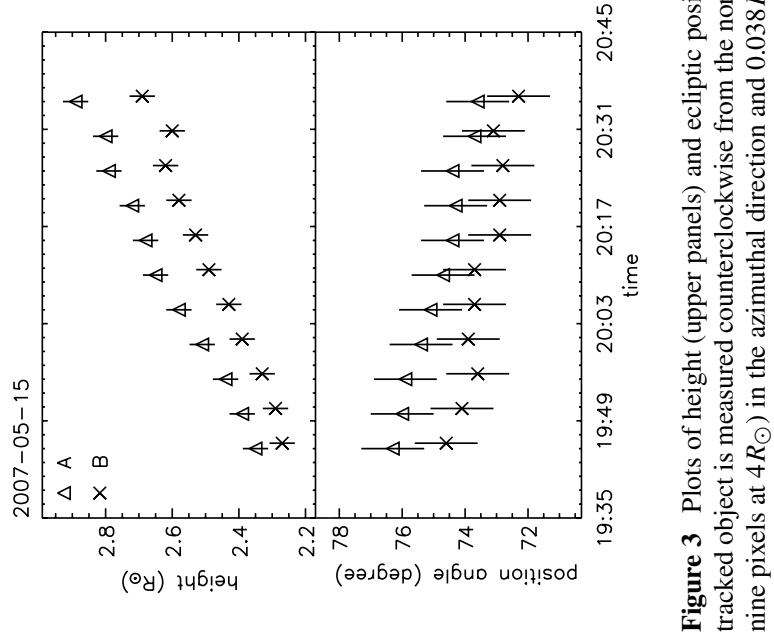



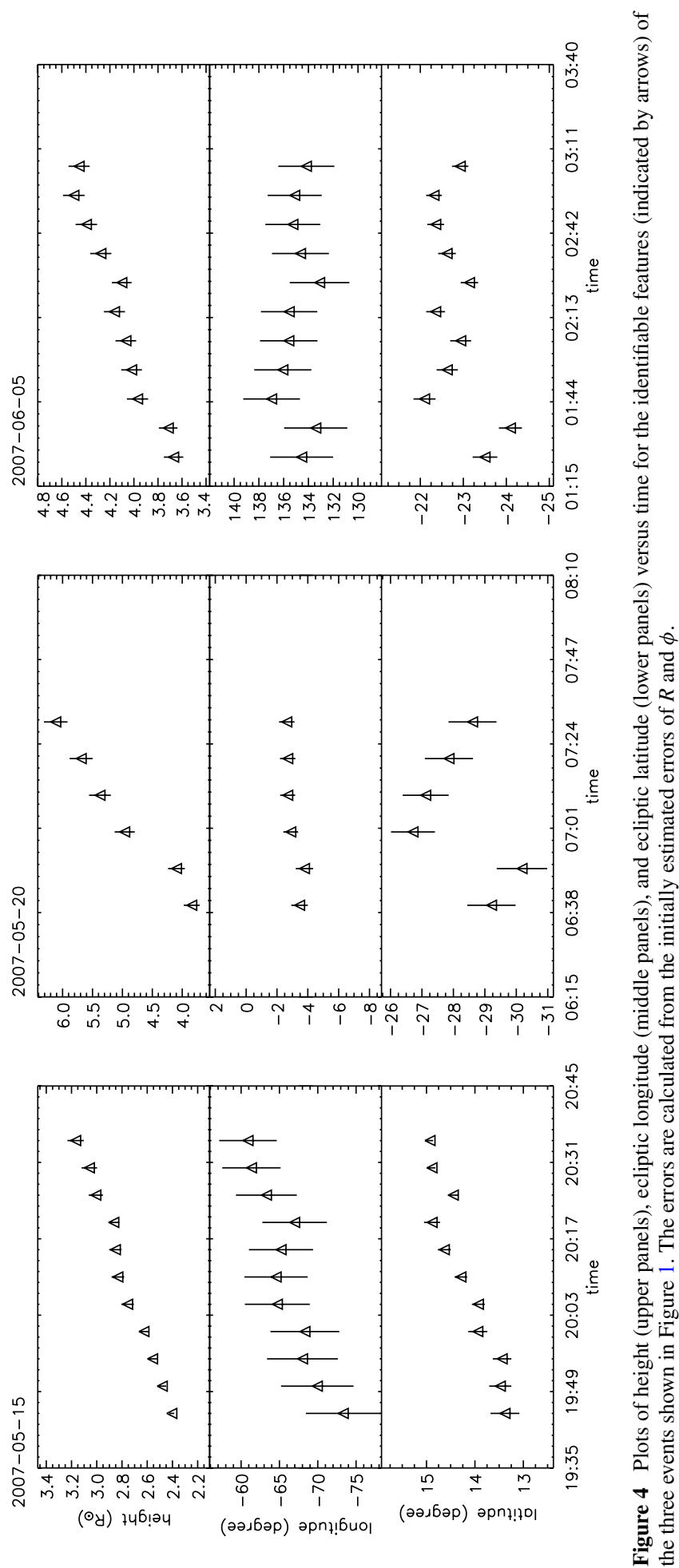
and ecliptic latitude suggest a radial motion of the tracked feature (i.e., at distances higher than $3.5 R_{\odot}$ no influence of the surrounding regions is observed). From HT plots and the EPA of the tracked feature we can infer whether the plasma is directed toward or away from the Earth. If we do not have a unique feature but just an extended CME front, we can still obtain HT diagrams by selecting an epipolar line (with $z$ chosen to be the same in A and B) and determining the intersection of the epipolar line with the front in the two images and measuring $R_{\mathrm{A}}$ and $\phi_{\mathrm{A}}$ and $R_{\mathrm{B}}$ and $\phi_{\mathrm{B}}$. The epipolar constraint $z=R_{\mathrm{B}} \cos \phi_{\mathrm{B}}=R_{\mathrm{A}} \cos \phi_{\mathrm{A}}$ is satisfied as a result of this construction.

We can also get the approximate position of the moving feature in a 3D coordinate system and the true radial speed of the feature. For the event on 15 May the projected speeds are $125 \mathrm{~km} \mathrm{~s}^{-1}$ (spacecraft A) and $99 \mathrm{~km} \mathrm{~s}^{-1}$ (spacecraft B). The true speed is $169 \mathrm{~km} \mathrm{~s}^{-1}$. On 20 May, the tracked feature moves with a speed of $548 \mathrm{~km} \mathrm{~s}^{-1}$ whereas the projected speeds measured in A and B spacecraft are 242 and $253 \mathrm{~km} \mathrm{~s}^{-1}$, respectively. The speed of the 5 June feature is around $94 \mathrm{~km} \mathrm{~s}^{-1}$ (with projected values of $78 \mathrm{~km} \mathrm{~s}^{-1}$ on image A and $67 \mathrm{~km} \mathrm{~s}^{-1}$ on image B). Note that for the CME on 20 May, which was directed toward the Earth, the real speed was double that of the projected values. Obtaining an estimate of the real speed values was not possible with the data from previous coronagraphs, without incorporating source locations from disk images such as those from the EIT. As a consequence, this simplified method applied to the SECCHI, in particular to COR1 data, may prove to be a useful tool for space weather forecasting. We should point out that one of our assumptions (i.e., affine geometry) depends on a ratio of the distance between the Sun and the object to the distance between the Sun and the observer. For our assumption to be valid that ratio should be small. Therefore this method cannot be applied to the heliospheric imager data.

Acknowledgements M.M. is grateful to the Goddard Space Flight Center for the financial support for her visit and for the facilities to carry out this work. M.M. and N.S. would like to acknowledge the Max Planck Institut für Sonnensystemforschung for the financial support for finalizing the work. The authors are grateful to Keith Feggans, Borut Podlipnik, and Nathan Rich for their help regarding the software. The authors thank the SECCHI/STEREO consortium for providing the data. The SECCHI data used here were produced by an international consortium of the Naval Research Laboratory (USA), Lockheed Martin Solar and Astrophysics Lab (USA), NASA Goddard Space Flight Center (USA), Rutherford Appleton Laboratory (UK), University of Birmingham (UK), Max-Planck-Institut for Solar System Research (Germany), Centre Spatiale de Liège (Belgium), Institut d'Optique Théorique et Appliquée (France), and Institut d'Astrophysique Spatiale (France).

\section{Appendix: Derivation of 3D Coordinates}

Under the assumptions made in the main text, the epipolar plane of an object is uniquely characterized by its distance $z$ from the Sun center and all epipolar planes are parallel to the ecliptic plane and have a common normal $\hat{\mathbf{z}}$. The value of $z$ can be directly retrieved for each point in an image. One of the constraints of the epipolar geometry is that the value of $z$ must be the same for corresponding pair of points in the two images $\mathrm{A}$ and $\mathrm{B}$, that is,

$$
z=R_{\mathrm{B}} \cos \phi_{\mathrm{B}}=R_{\mathrm{A}} \cos \phi_{\mathrm{A}} .
$$

Here, $R_{\mathrm{B}}$ and $R_{\mathrm{A}}$ are the distances from the Sun center as measured in an HT diagram and $\phi_{\mathrm{B}}$ and $\phi_{\mathrm{A}}$ are the respective position angles measured from ecliptic north counterclockwise (see Figure 2, left panel). Equation (2) can be verified from Figure 3: For the CME of 15 May, at east limb (EPA around $\left.90^{\circ}\right) z$ is around 0 for both A and B images. For the CME on 20 May, seen at the South Pole (EPA around $180^{\circ}$ ), the distance from the Sun center to 
the corresponding features observed in $\mathrm{A}$ and $\mathrm{B}$ images are equal (i.e., $z_{\mathrm{A}}$ and $z_{\mathrm{B}}$ are equal). For the CME on 5 June, seen at the southwest limb (EPA around $245^{\circ}$ ), both $R$ and $\phi$ are different, but $z$ is approximately the same for A and B images. This demonstrates that we follow the same feature in the two images.

The 3D position of an object on the epipolar plane $z$ is then

$$
\mathbf{R}_{3 \mathrm{D}}=\mathbf{R}_{2 \mathrm{D}}+z \hat{\mathbf{z}},
$$

where the position $\mathbf{R}_{2 \mathrm{D}}$ in the epipolar plane relative to the projection of the Sun's center onto the plane could be written as

$$
\mathbf{R}_{2 \mathrm{D}}=\alpha \hat{\mathbf{l}}_{\mathrm{A}}+\beta \hat{\mathbf{l}}_{\mathrm{B}}
$$

Here, $\alpha$ and $\beta$ are parameters to be determined.

The projection of our object on image plane $\mathrm{B}$ (i.e., along $\hat{\mathbf{P}}_{\mathrm{B}}=\hat{\mathbf{l}}_{\mathrm{B}} \times \hat{\mathbf{z}}$ ) is represented by the thick solid arrow in the right-hand-side panel of Figure 2 and it has a value of $-R_{\mathrm{B}} \sin \left(\phi_{\mathrm{B}}\right)$. This can be easily inferred from the left-hand-side panel of Figure 2, which shows the view in the plane of image $\mathrm{B}$. Similarly, its projection on image $\mathrm{A}$ (i.e., along $\left.\hat{\mathbf{P}}_{\mathrm{A}}=\hat{\mathbf{z}} \times \hat{\mathbf{l}}_{\mathrm{A}}\right)$ is $-R_{\mathrm{A}} \sin \left(\phi_{\mathrm{A}}\right)$ Multiplying Equation (4) with $\hat{\mathbf{P}}_{\mathrm{A}}$ and $\hat{\mathbf{P}}_{\mathrm{B}}$ we obtain the values for $\alpha$ and $\beta$ if we make use of the relations $\hat{\mathbf{l}}_{\mathrm{B}} \cdot \hat{\mathbf{l}}_{\mathrm{A}}=\cos \gamma$ and $\hat{\mathbf{l}}_{\mathrm{B}} \times \hat{\mathbf{l}}_{\mathrm{A}}=\hat{\mathbf{z}} \sin \gamma$. The position of the object in the epipolar plane is then

$$
\mathbf{R}_{2 \mathrm{D}}=\frac{R_{\mathrm{B}} \sin \phi_{\mathrm{B}} \hat{\mathbf{l}}_{\mathrm{A}}-R_{\mathrm{A}} \sin \phi_{\mathrm{A}} \hat{\mathbf{l}}_{\mathrm{B}}}{\sin \gamma},
$$

and the magnitude of this vector is $R_{2 \mathrm{D}}=\sqrt{\alpha^{2}+\beta^{2}+2 \alpha \beta \cos \gamma}$.

Knowing $\mathbf{R}_{2 \mathrm{D}}$ and the distance of the epipolar plane with respect to the Sun center $(z)$ we can get the position of our moving feature in a $3 \mathrm{D}$ coordinate system:

$$
\mathbf{R}_{3 \mathrm{D}}=\frac{R_{\mathrm{B}} \sin \phi_{\mathrm{B}} \hat{\mathbf{l}}_{\mathrm{A}}-R_{\mathrm{A}} \sin \phi_{\mathrm{A}} \hat{\mathbf{l}}_{\mathrm{B}}}{\sin \gamma}+z \hat{\mathbf{z}},
$$

with a magnitude of

$$
R_{3 \mathrm{D}}=\sqrt{\left(R_{2 \mathrm{D}}\right)^{2}+z^{2}} .
$$

The ecliptic latitude (see Thompson (2006) and references therein for a detailed description of the coordinate systems) is obviously given by

$$
\theta=\arctan \frac{R_{2 \mathrm{D}}}{z},
$$

which is unique within the allowed latitude range $[-\pi / 2, \pi / 2]$.

Supposing that the Earth is situated at equal angles between STEREO A and B we can obtain the difference $\Delta \lambda$ in ecliptic longitude of the object from the Earth by evaluating

$$
\tan \Delta \lambda=\frac{\mathbf{R}_{2 \mathrm{D}} \cdot\left(\hat{\mathbf{l}}_{\mathrm{B}}-\hat{\mathbf{l}}_{\mathrm{A}}\right) /\left|\left(\hat{\mathbf{l}}_{\mathrm{B}}-\hat{\mathbf{l}}_{\mathrm{A}}\right)\right|}{\mathbf{R}_{2 \mathrm{D}} \cdot\left(\hat{\mathbf{l}}_{\mathrm{B}}+\hat{\mathbf{l}}_{\mathrm{A}}\right) /\left|\left(\hat{\mathbf{l}}_{\mathrm{B}}+\hat{\mathbf{l}}_{\mathrm{A}}\right)\right|} .
$$

The result is

$$
\Delta \lambda=\arctan \left[\tan \left(\frac{\gamma}{2}\right) \frac{a-b}{a+b}\right],
$$


Table 1 Angular ranges of $\Delta \lambda$ calculated from Equation (10).

\begin{tabular}{lc}
\hline Cases & Longitude \\
\hline$a-b>0$ & {$\left[-\frac{\pi}{2}, 0\right]$} \\
$a+b<0$ & \\
$a-b>0$ & {$\left[-\pi,-\frac{\pi}{2}\right]$} \\
$a+b>0$ & \\
$a-b<0$ & {$\left[\frac{\pi}{2}, \pi\right]$} \\
$a+b>0$ & \\
$a-b<0$ & {$\left[0, \frac{\pi}{2}\right]$} \\
$a+b<0$ &
\end{tabular}

where $a=-R_{\mathrm{A}} \sin \phi_{\mathrm{A}}$ and $b=R_{\mathrm{B}} \sin \phi_{\mathrm{B}}$. As the ecliptic longitude ranges between $-\pi$ and $\pi$, Equation (10) does not yield a unique value. Depending on the signs of the numerator and the denominator in the arctangent of Equation (10), $\Delta \lambda$ falls into the angular ranges listed in Table 1.

\section{References}

Aschwanden, M.J., Burlaga, L.F., Kaiser, M.L., Ng, C.K., Reames, D.V., Reiner, M.J., et al.: 2008, Space Sci. Rev. 136, 565.

Brueckner, G.E., Howard, R.A., Koomen, M.J., Korendyke, C.M., Michels, D.J., Moses, J.D., et al.: 1995, Solar Phys. 162, 357.

Cremades, H., Bothmer, V.: 2004, Astron. Astrophys. 422, 307.

Dere, K.P., Wang, D., Howard, R.: 2005, Astrophys. J. 620, L119.

Feng, L., Inhester, B., Solanki, S., Wiegelmann, T., Podlipnik, B., Howard, R.A., Wuelser, J.-P.: 2007, Astrophys. J. 671, L205.

Howard, R.A., Moses, J.D., Vourlidas, A., Newmark, J.S., Socker, D.G., Plunkett, S.P., et al.: 2008, Space Sci. Rev. 136, 67.

Inhester, B.: 2006, Publ. Int. Space Sci. Inst., astro-ph/0612649, to appear.

Low, B.C., Hundhausen, A.J.: 1987, J. Geophys. Res. 92, 2221.

Michalek, G.: 2006, Solar Phys. 237, 101.

Michalek, G., Gopalswamy, N., Yashiro, S.: 2003, Astrophys. J. 584, 472.

Moran, T.G., Davila, J.: 2004, Science 305, 66.

Pizzo, V.J., Biesecker, D.A.: 2004, Geophys. Res. Lett. 31, L21802.

Sheeley, N.R. Jr., Walters, J.H., Wang, Y.-M., Howard, R.A.: 1999, J. Geophys. Res. 104, 24739.

Srivastava, N., Schwenn, R., Inhester, B., Martin, S.F., Hanaoka, Y.: 2000, Astrophys. J. 534, 468.

St. Cyr, O.C., Burkepile, J.T., Hundhausen, A.J., Lecinski, A.R.: 1999, J. Geophys. Res. 104, 12493.

Thernisien, A.F.R., Howard, R.A., Vourlidas, A.: 2006, Astrophys. J. 652, 763.

Thompson, W.T.: 2006, Astron. Astrophys. 449, 791.

Thompson, W.T., Davila, J.M., Fisher, R.R., Orwig, L.E., Mentzell, J.E., Hetherington, S.E., 2003, In: Keil, S.L., Avakyan, S.V. (eds.) Innovative Telescopes and Instrumentation for Solar Astrophysics, Proc. SPIE 4853, 1 .

Xie, H., Ofman, L., Lawrence, G.: 2004, J. Geophys. Res. 109, A03109.

Yashiro, S., Gopalswamy, M., Michalek, G., St. Cyr, O.C., Plunkett, S.P., Rich, N.B., Howard, R.A.: 2004, J. Geophys. Res. 109, A07105.

Zhao, X.P., Plunkett, S.P., Liu, W.: 2002, J. Geophys. Res. 107, 1223. 